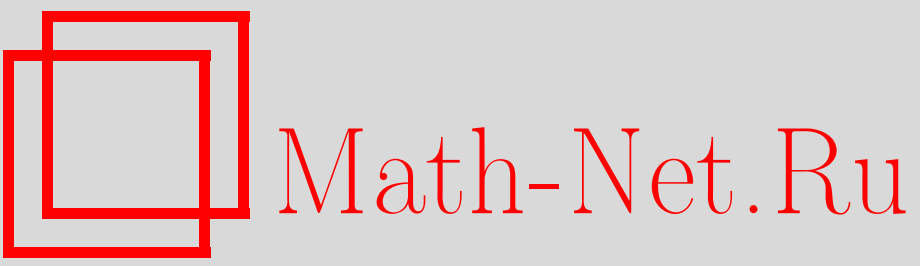

М. Е. Палистрант, Влияние немагнитной примеси на сверхпроводимость в двухзонных системах с переменной плотностью носителей заряда, ТМФ, 1997, том 111, номер 2, 289-303

DOI: https://doi.org/10.4213/tmf1008

Использование Общероссийского математического портала Math-Net.Ru подразумевает, что вы прочитали и согласны с пользовательским соглашением

http://www.mathnet.ru/rus/agreement

Параметры загрузки:

IP : 54.80 .97 .219

26 апреля 2023 г., 13:46:13 
ТЕОРЕТИЧЕСКАЯ

И МАТЕМАТИЧЕСКАЯ

ФИЗИКА

Том 111, № 2

май, 1997

М.Е. Палистрант*

\title{
ВЛИЯНИЕ НЕМАГНИТНОЙ ПРИМЕСИ НА СВЕРХПРОВОДИМОСТЬ В ДВУХЗОННЫХ СИСТЕМАХ С ПЕРЕМЕННОЙ ПЛОТНОСТЬЮ НОСИТЕЛЕЙ ЗАРЯДА
}

\begin{abstract}
С помощью теории возмущений получены аналитические выражения для температуры сверхпроводящего перехода $T_{C}$ и параметров порядка $\Delta_{n}(n=1,2)$ при различных значениях плотности носителей заряда (химического потенциала $\mu$ ) в двухзонных сверхпроводниках с немагнитной примесью. При введении примесей в систему возможны два механизма ее влияния на величины $T_{C}$ и $\Delta_{n}$ : изменение величины $\mu$ и возникновение межзонного рассеяния на примесном потенциале. Эти механизмы могут быть либо конкурирующими, либо оба способствовать разрушению сверхпроводимости. Существенную роль при этом играет фактор заполнения энергетических зон, благодаря которому сверхпроводимость может полностью подавляться немагнитной примесью.
\end{abstract}

\section{1. ВВЕДЕНИЕ}

В соответствии с теоремой Андерсона [1] и результатами Абрикосова и Горькова [2] немагнитная примесь не влияет на термодинамические свойства обычных изотропных сверхпроводников. Учет анизотропии матричного элемента электрон-электронного взаимодействия нарушает теорему Андерсона и приводит к зависимости температуры сверхпроводящего перехода $T_{C}$ от концентрации примеси [3]. Наряду с этим важную роль в определении свойств анизотропных сверхпроводников играют особенности в электронном энергетическом спектре. Речь идет о перекрытии энергетических полос на поверхности Ферми и наличии особенностей Ван-Хова-Лифшица.

Как показано в наших работах [4] и монографии [5], немагнитная примесь значительно влияет на критическую температуру и вообше на термодинамические свойства многозонных сверхпроводников благодаря межзонному каналу рассеяния электронов на немагнитной примеси.

Немагнитная примесь влияет также на термодинамические свойства сверхпроводника при электронных топологических переходах. Особый интерес в настояшее время представляют так называемые протяженные особенности Ван-Хова, когда плотность электронных состояний имеет расходимость в нуле порядка $E^{-1 / 4}$ или $E^{-1 / 2}(E-$ энергия, отсчитанная от особенностей Ван-Хова) [6].

* Институт прикладной физики АН Молдовы, Кишинев, Молдова 
Отметим, что влияние особенностей типа $E^{-1 / 2}$ на термодинамические и кинетические свойства сверхпроводников исследовалось нами еше задолго до открытия высокотемпературной сверхпроводимости (ВТСП) [7, 8]. В частности, было показано, что рассеяние электронов на примесном потенциале в сверхпроводниках с такой особенностью в плотности электронных состояний понижает температуру сверхпроводяшего перехода. Открытие материалов, обладающих свойством ВТСП [9], и многочисленные экспериментальные исследования показали, что в таких сложных анизотропных системах как $\mathrm{La}_{2} \mathrm{CuO}_{4}$ и $\mathrm{YBa}_{2} \mathrm{Cu}_{3} \mathrm{O}_{7-\delta}$ температура перехода в сверхпроводящее состояние $T_{C}$ существенно зависит от концентраций кислорода и немагнитной примеси. В работах [10] мы попытались объяснить убывание величины $T_{C}$ с ростом неупорядочения кислорода, а также при замешении атомов меди немагнитной примесью ( $\mathrm{Al}, \mathrm{Zn}$ и др.) на основе двухзонной модели $[11,12]$. Это убывание определяется механизмом межзонного рассеяния электронов на кислороде (примеси).

Отметим, что, помимо приведенных выше, имеются и другие механизмы, приводящие к убыванию величины $T_{C}$ с ростом концентрации примеси в материалах ВТСП. Так, например, в работах $[13,14]$ в основе такого убывания лежит эффект неупорядочения на кулоновском псевдопотенциале и на магнитных корреляциях, вводимый немагнитной примесью.

В настоящее время проблема влияния немагнитной примеси ( $\mathrm{Zn}, \mathrm{Al}, \mathrm{Ga})$ на температуру сверхпроводяшего перехода является одним из важных направлений в исследовании сверхпроводящих свойств материалов ВТСП. Решение ее может пролить свет на механизмы ВТСП. Дело в том, что различные немагнитные примеси по-разному влияют на величину $T_{C}$ в купратных соединениях. Замещение атомов $\mathrm{Cu}$ атомами $\mathrm{Al}$ или $\mathrm{Ga}$ слабо разрушает сверхпроводимость, в то время как допирование цинком приводит к быстрому подавлению сверхпроводимости.

Слабое влияние немагнитной примеси $(\mathrm{Al}, \mathrm{Ga})$ на величину $T_{C}$ можно объяснить, если анизотропия матричного элемента электрон-электронного взаимодействия мала [3] или если мало межзонное рассеяние на примесном потенциале $[4,10]$. Атомы же цинка, замешая атомы меди, ведут себя как магнитная примесь, подавляя сверхпроводимость. Однако экспериментальные исследования [15] показывают, что в соединении $\mathrm{YBa}_{2} \mathrm{Cu}_{3} \mathrm{O}_{7}$, допированном цинком, отсутствует магнитное разрушение электронных пар. Следовательно, теория Абрикосова и Горькова влияния парамагнитной примеси на сверхпроводимость [2] не может объяснить подавление сверхпроводимости в таких системах.

Наши исследования $[4,10]$, основанные на двухзонной модели, позволяют объяснить довольно быстрое убывание величины $T_{C}$ с ростом концентрации примеси. Однако в этой модели немагнитная примесь не может полностью подавить сверхпроводимость.

Предложенная в $[4,10]$ теория сверхпроводимости основана на механизме электрон-фононного взаимодействия и не предполагает пониженной плотности носителей заряда. В случае нефононного механизма сверхпроводимости или фононного механизма с пониженной плотностью носителей, что имеет место в материалах ВТСП, величина $T_{C}$ и другие характеристики зависят от значения химического потенциала (плотности носителей заряда) [16-19]. При этом важную роль играет последовательное заполнение электронами перекрываюшихся на поверхности Ферми энергетических зон. В частности, оказывается возможным получить наблюдаемую на эксперименте ступенчатую зависимость величины $T_{C}$ от плотности носителей заряда в двухзонной модели при элект- 
ронном топологическом переходе [20] или в трехзонной модели [21]. В [16-21] были рассмотрены упорядоченные системы. В двухзонных системах с хаотически распределенной примесью необходимо учитывать внутризонные и межзонные рассеяния электронов на примесном потенциале.

Данная работа посвящена исследованию сверхпроводимости в двухзонных примесных системах с переменной плотностью носителей заряда при нефононном механизме сверхпроводимости. Во втором разделе приведены выражения для одночастичных функций Грина (нормальной и аномальной), усредненных по хаотически распределенной примеси, а также основные уравнения теории сверхпроводимости. В третьем разделе получена система уравнений для параметров порядка $\Delta_{n}(n=1,2)$ вблизи температуры сверхпроводяшего перехода в области малых и больших концентраций примеси. Четвертый раздел посвящен определению температуры сверхпроводяшего перехода в области малых и больших концентраций примеси при определенных значениях химического потенциала $\mu$. В разделе пятом приведены решения системы уравнений для параметров порядка $\Delta_{1}$ и $\Delta_{2}$ при заданных конкретных значениях химического потенциала $\mu$ и $T=0$. В последнем разделе обсуждаются полученные результаты и делаются попытки объяснить имеющиеся экспериментальные данные по влиянию немагнитной примеси на $T_{C}$.

\section{2. ВЫРАЖЕНИЯ ДЛЯ ФУНКЦИЙ ГРИНА И ОСНОВНЫЕ УРАВНЕНИЯ}

Гамильтониан двухзонной системы с хаотически распределенной примесью представим в виде

$$
H=H_{0}+H_{\mathrm{imp}}
$$

где $H_{0}$ - модельньй гамильтониан чистой двухзонной системы [11], a $H_{\mathrm{imp}}$ - оператор взаимодействия электронов с хаотически распределенной примесью [4].

На основании теории возмущений [22], рассматривая $H_{\mathrm{imp}}$ как возмушение, для нормальной и аномальной функций Грина, усредненных по положениям хаотически распределенной примеси, получаем соответственно

$$
G_{n}(\vec{p}, \omega)=-\frac{i \widetilde{\omega}_{n}+\tilde{\varepsilon}_{n}}{\widetilde{\omega}_{n}^{2}+\tilde{\varepsilon}_{n}^{2}+\widetilde{\Delta}_{n}^{2}}, \quad F_{n}(\vec{p}, \omega)=\frac{\widetilde{\Delta}_{n}}{\widetilde{\omega}_{n}^{2}+\widetilde{\varepsilon}_{n}^{2}+\widetilde{\Delta}_{n}^{2}}
$$

где

$$
\widetilde{\omega}_{n}=\omega+\operatorname{Im} M_{n}^{*}(\omega), \quad \widetilde{\Delta}_{n}=\bar{\Delta}_{n}+\Sigma_{n}(\omega), \quad \tilde{\varepsilon}_{n} \approx \varepsilon_{n}+\operatorname{Re} M_{n}^{*}(0)
$$

здесь

$$
\begin{aligned}
& M_{n}(\omega)=-c \sum_{m} \sum_{\vec{k}}\left|V\left(\vec{p}_{n F}-\vec{k}_{m F}\right)\right|^{2}\left|\chi\left(n \vec{p}_{n F}, m \vec{k}_{m F}\right)\right|^{2} G_{m}(\vec{k}, \omega), \\
& \Sigma_{n}(\omega)=c \sum_{m} \sum_{\vec{k}}\left|V\left(\vec{p}_{n F}-\vec{k}_{m F}\right)\right|^{2}\left|\chi\left(n \vec{p}_{n F}, m \vec{k}_{m F}\right)\right|^{2} F_{m}(\vec{k}, \omega),
\end{aligned}
$$

$c$ - концентрация примеси, $V\left(\vec{p}_{n F}-\vec{k}_{m F}\right)$ - фурье-образ потенциала рассеяния на примеси, $\chi\left(n \vec{p}_{n F}, m \vec{k}_{m F}\right)$ - интеграл от амплитуд Блоха по объему элементарной ячейки, $\mu$ - химический потенциал; $n, m=1,2$. 
Предполагаем простой закон дисперсии

$$
\xi_{n}(\vec{p})=\zeta_{n}+\frac{p^{2}}{2 m_{n}}, \quad \varepsilon_{n}(\vec{p})=\xi_{n}(\vec{p})-\mu .
$$

Перейдем в формулах (4) от суммирования по $\vec{k}$ к интегрированию по $\tilde{\varepsilon}$ согласно соотношению

$$
\sum_{\vec{k}}|V(\vec{p}-\vec{k})|^{2}|\chi(n \vec{p}, m \vec{k})|^{2} \Phi\left(\tilde{\varepsilon}_{m}\right)=\frac{1}{2 \tau_{n m}} \frac{1}{\pi} \int_{-D_{m}}^{D_{c m}} d \tilde{\varepsilon}_{m} \Phi\left(\tilde{\varepsilon}_{m}\right),
$$

где

$$
\begin{gathered}
\frac{1}{2 \tau_{n m}}=\frac{c}{8 \pi^{2}} \int \frac{d S_{m}}{\left|\nabla \varepsilon_{m}\right|}\left|V\left(\vec{p}_{n F}-\vec{k}_{m F}\right)\right|^{2}\left|\chi\left(n \vec{p}_{n F}, m \vec{k}_{m F}\right)\right|^{2} \\
D_{c m}=\zeta_{c m}^{\prime}-\mu, \quad D_{m}=\mu-\zeta_{m}^{\prime}, \quad \zeta_{c m}^{\prime}=\zeta_{c m}+\operatorname{Re} M_{m}^{*}(0), \quad \zeta_{m}^{\prime}=\zeta_{m}+\operatorname{Re} M_{m}^{*}(0)
\end{gathered}
$$

$\zeta_{c m}$ - энергия обрезания интегралов по энергии, $\tau_{n m}-$ времена релаксации рассеяния на примеси.

Выполнив интегрирование в формулах (4) и воспользовавшись соотношениями (3), получаем

$$
\begin{aligned}
& \widetilde{\omega}_{n}=\omega+\sum_{m} \frac{1}{2 \tau_{n m}} \frac{\widetilde{\omega}_{m} f_{m}}{\sqrt{\widetilde{\omega}_{m}^{2}+\widetilde{\Delta}_{m}^{2}}} \\
& \widetilde{\Delta}_{n}=\bar{\Delta}_{n}+\sum_{m} \frac{1}{2 \tau_{n m}} \frac{\widetilde{\Delta}_{m} f_{m}}{\sqrt{\widetilde{\omega}_{m}^{2}+\widetilde{\Delta}_{m}^{2}}}
\end{aligned}
$$

где

$$
f_{m}=\frac{1}{\pi}\left[\operatorname{arctg} \frac{D_{c m}}{\sqrt{\widetilde{\omega}_{m}^{2}+\widetilde{\Delta}_{m}^{2}}}+\operatorname{arctg} \frac{D_{m}}{\sqrt{\widetilde{\omega}_{m}^{2}+\widetilde{\Delta}_{m}^{2}}}\right] .
$$

Систему уравнений на параметры порядка $\Delta_{n}(n=1,2)$ можно привести к виду

$$
\begin{aligned}
\bar{\Delta}_{n}= & \sum_{l} N_{l} V_{n l} \bar{\Delta}_{l} \int_{-D_{l}}^{D_{c l}} d \varepsilon_{l} \frac{\operatorname{th} \frac{\beta}{2} \sqrt{\tilde{\varepsilon}_{l}^{2}+\bar{\Delta}_{l}^{2}}}{2 \sqrt{\tilde{\varepsilon}_{l}^{2}+\bar{\Delta}_{l}^{2}}}+ \\
& +\sum_{l} V_{n l} N_{l} \frac{2 \pi}{\beta} \sum_{\omega>0}\left[\frac{\widetilde{\Delta}_{l} f_{l}}{\sqrt{\widetilde{\omega}_{l}^{2}+\widetilde{\Delta}_{l}^{2}}}-\frac{\bar{\Delta}_{l} f_{l}^{0}}{\sqrt{\omega^{2}+\bar{\Delta}_{l}^{2}}}\right]
\end{aligned}
$$

здесь $f_{l}^{0}=\left.f_{l}\right|_{\widetilde{\omega}=\omega, \tilde{\Delta}=\bar{\Delta}}, V_{n m}$ - константы внутризонного и межзонного взаимодействий, $N_{n}$ - плотность электронных состояний на $n$-й полости поверхности Ферми. Обозначив $u_{n}=\frac{\widetilde{\omega}_{n}}{\widetilde{\Delta}_{n}}$, систему уравнений $(9)$ можно привести к виду

$$
\begin{gathered}
u_{1} \pm \alpha_{1} f_{2} \frac{u_{1}-u_{2}}{\sqrt{1+u_{2}^{2}}}=\frac{\omega}{\bar{\Delta}_{1}}, \quad u_{2}+\alpha_{2} f_{1} \frac{u_{2}-u_{1}}{\sqrt{1+u_{1}^{2}}}=\frac{\omega}{\bar{\Delta}_{2}} \\
\alpha_{1}=\frac{1}{2 \tau_{12} \bar{\Delta}_{1}}, \quad \alpha_{2}=\frac{1}{2 \tau_{21} \bar{\Delta}_{2}}, \quad \omega>0 .
\end{gathered}
$$


При этом система уравнений (11) перепишется следующим образом:

$$
\begin{aligned}
\bar{\Delta}_{n}= & \sum_{l} V_{n l} N_{l} \bar{\Delta}_{l} \int_{-D_{l}}^{D_{c l}} d \tilde{\varepsilon}_{l} \frac{\operatorname{th} \frac{\beta}{2} \sqrt{\tilde{\varepsilon}_{l}^{2}+\bar{\Delta}_{l}^{2}}}{2 \sqrt{\tilde{\varepsilon}_{l}^{2}+\bar{\Delta}_{l}^{2}}}+ \\
& +V_{n 1} N_{1} \frac{2 \pi}{\beta} \sum_{\omega>0} f_{1}\left[\frac{1}{\sqrt{u_{1}^{2}+1}}-\frac{\bar{\Delta}_{1}}{\sqrt{\omega^{2}+\bar{\Delta}_{1}^{2}}}\right] \pm \\
& \pm V_{n 2} N_{2} \frac{2 \pi}{\beta} \sum_{\omega>0} f_{2}\left[\frac{1}{\sqrt{u_{2}^{2}+1}} \mp \frac{\bar{\Delta}_{2}}{\sqrt{\omega^{2}+\bar{\Delta}_{2}^{2}}}\right] .
\end{aligned}
$$

Верхний знак операции $( \pm)$ или $(\mp)$ в уравнениях $(12)$ и (13) соответствует $\widetilde{\Delta}_{1}, \widetilde{\Delta}_{2}>0$, а нижний $-\widetilde{\Delta}_{1}>0, \widetilde{\Delta}_{2}<0$. Из-за сложной зависимости величины $f_{m}(10)$ от $\widetilde{\omega}_{m}$ и $\widetilde{\Delta}_{m}$ решить систему уравнений (12) и (13) в обшем виде не представляется возможным. В дальнейшем мы будем рассматривать области слабопониженной концентрации носителей, когда выполняются соотношения $D_{l} / \Delta_{l} ; D_{c l} / \bar{\Delta}_{l} \gg 1$, а также отдельные точки $D_{l}=0$ и $D_{c l}=0(l=1,2)$. В этих случаях можно считать $f_{l}=f_{l}^{0}=$ const.

\section{3. ОБЛАСТЬ ТЕМПЕРАТУР, БЛИЗКИХ К $T_{C}$}

При $T \sim T_{C}$ ограничимся в уравнении $(13)$ линейными по $\bar{\Delta}_{n}$ членами и представим это уравнение в случае $\bar{\Delta}_{1}>0$ и $\bar{\Delta}_{2}>0$ в виде

$$
\bar{\Delta}_{n}=\sum_{l} V_{n l} N_{l} \bar{\Delta}_{l} \widetilde{\xi}_{l}+\sum_{l} V_{n l} N_{l} \frac{2 \pi}{\beta} \sum_{\omega>0} f_{l}\left[\frac{1}{\sqrt{u_{l}^{2}+1}}-\frac{\bar{\Delta}_{l}}{\omega}\right],
$$

где

$$
\widetilde{\xi}_{l}=\frac{1}{2} \int_{-D_{l}}^{D_{c l}} d \tilde{\varepsilon}_{l} \frac{\operatorname{th} \frac{\beta \tilde{\varepsilon}_{l}}{2}}{\tilde{\varepsilon}_{l}} .
$$

Мы имеем также при $\bar{\Delta}_{n} \rightarrow 0$ соотношение $u_{n} \gg 1$. Воспользуемся в дальнейшем методикой расчета, предложенной в работах [5, 23] при исследовании термодинамических свойств обычных двухзонных сверхпроводников.

Ишем решения системы уравнений (12) в виде

$$
u_{n}=u_{n}^{0}+u_{n}^{\prime},
$$

где $u_{n}^{\prime} \ll u_{n}^{0}$, а $u_{1}^{0}$ и $u_{2}^{0}$ удовлетворяют системе уравнений

$$
u_{1}^{0}+\alpha_{1} f_{2} \frac{u_{1}^{0}-u_{2}^{0}}{u_{2}^{0}}=\frac{\omega}{\bar{\Delta}_{1}}, \quad u_{2}^{0}+\alpha_{2} f_{1} \frac{u_{2}^{0}-u_{1}^{0}}{u_{1}^{0}}=\frac{\omega}{\bar{\Delta}_{2}} .
$$

Решения (17) имеют вид

$$
u_{1}^{0}=\frac{\omega}{\bar{\Delta}_{1}} \frac{\frac{\omega}{\bar{\Delta}_{2}}+\alpha_{1} f_{2} \bar{z}+\alpha_{2} f_{1}}{\frac{\omega}{\bar{\Delta}_{2}}+\alpha_{1} f_{2}+\alpha_{2} f_{1}}, \quad \bar{z}=\frac{\bar{\Delta}_{1}}{\bar{\Delta}_{2}},
$$


$u_{2}^{0}$ получаем из $(18)$ заменой индексов $1 \rightleftarrows 2$. Подставляя (16) в (12) и учитывая (18) и условие $u_{n}^{\prime} \ll u_{n}^{0}$, находим

$$
u_{1}^{\prime}=\frac{\alpha_{1} f_{2}\left(1-\frac{u_{2}^{0}}{u_{1}^{0}}\right)\left[u_{1}^{0}+\alpha_{2} f_{1}\left(1-\frac{u_{2}^{0}}{u_{1}^{0}}\right)\right]}{2\left(u_{2}^{0}\right)^{2}\left[u_{2}^{0}+\alpha_{1} f_{2}+\frac{u_{2}^{0}}{u_{1}^{0}} \alpha_{2} f_{1}\right]}
$$

$u_{2}^{\prime}$ получается из (19) заменой индексов $1 \rightleftarrows 2$. Выполнив далее в уравнении (14) разложение по $1 / u_{l} \ll 1$, приведем его к виду

$$
\bar{\Delta}_{n}=\sum_{l} V_{n l} N_{l} \bar{\Delta}_{l} \widetilde{\xi}_{l}+\frac{2 \pi}{\beta} \sum_{l} V_{n l} N_{l} f_{l} \sum_{\omega>0}\left(\frac{1}{u_{l}^{0}}-\frac{\bar{\Delta}_{l}}{\omega}-\frac{1}{2\left(u_{l}^{0}\right)^{3}}-\frac{u_{l}^{\prime}}{\left(u_{l}^{0}\right)^{2}}\right) .
$$

Подставим решения (18) и (19) в (20) и выполним суммирование по $\omega$ аналогично тому, как это сделано в [5].

Приведем результаты для случая малых и больших концентраций примеси.

А. Малые концентрации примеси $\rho_{1}=(1 / 2) \tau_{12} \pi T \ll 1$ и $\rho_{2}=12 \tau_{21} \pi T \ll 1$. В этом случае система уравнений для параметров порядка $\bar{\Delta}_{n}$ может быть приведена к виду

$$
\begin{aligned}
\bar{\Delta}_{n}= & \sum_{l} V_{n l} N_{l} \bar{\Delta}_{l} \widetilde{\xi}_{l}+N_{1} V_{n 1} f_{1}\left\{\frac{N_{2} f_{2}}{N_{1} f_{1}+N_{2} f_{2}}\left(\bar{\Delta}_{2}-\bar{\Delta}_{1}\right) \frac{\pi^{2}}{4} \rho^{\prime}-\right. \\
& -\bar{\Delta}_{2}^{2}\left(\bar{\Delta}_{2}-\bar{\Delta}_{1}\right) \frac{N_{2} f_{2}}{N_{1} f_{1}+N_{2} f_{2}} \frac{\beta^{2} \pi^{2}}{96} \rho^{\prime}- \\
& \left.-\frac{7 \zeta(3)}{8 \pi^{2}} \beta^{2} \bar{\Delta}_{1}^{3}\left[1-\frac{\pi^{4}\left(\bar{\Delta}_{2}-\bar{\Delta}_{1}\right)}{\bar{\Delta}_{1} 28 \zeta(3)} \frac{N_{2} f_{2}}{N_{1} f_{1}+N_{2} f_{2}} \rho^{\prime}\right]\right\}+ \\
& +N_{2} V_{n 2} f_{2}\left\{\frac{N_{1} f_{1}}{N_{1} f_{1}+N_{2} f_{2}}\left(\bar{\Delta}_{1}-\bar{\Delta}_{2}\right) \frac{\pi^{2}}{4} \rho^{\prime}-\right. \\
& -\bar{\Delta}_{1}^{2}\left(\bar{\Delta}_{1}-\bar{\Delta}_{2}\right) \frac{N_{1} f_{1}}{N_{1} f_{1}+N_{2} f_{2}} \frac{\beta^{2} \pi^{2}}{96} \rho^{\prime}- \\
& \left.-\frac{7 \zeta(3)}{8 \pi^{2}} \beta^{2} \bar{\Delta}_{2}^{3}\left[1+\frac{\pi^{4}\left(\bar{\Delta}_{1}-\bar{\Delta}_{2}\right)}{\bar{\Delta}_{2} 28 \zeta(3)} \frac{N_{1} f_{1}}{N_{1} f_{1}+N_{2} f_{2}} \rho^{\prime}\right]\right\}
\end{aligned}
$$

где

$$
\rho^{\prime}=f_{2} \rho_{1}+f_{1} \rho_{2}
$$

Б. Большие конщентрации примеси $\rho_{1} \gg 1$ и $\rho_{2} \gg 1$. Тогда система уравнений на параметры порядка $\bar{\Delta}_{n}$ после суммирования по $\omega$ приобретает вид

$$
\begin{aligned}
\bar{\Delta}_{n}= & \sum_{l} V_{n l} \bar{\Delta}_{l} \widetilde{\xi}_{l}+N_{1} V_{n 1} f_{1}\left\{\frac{N_{2} f_{2}}{N_{1} f_{1}+N_{2} f_{2}}\left(\bar{\Delta}_{2}-\bar{\Delta}_{1}\right) \ln 2 \gamma \rho^{\prime}-\frac{\beta^{2}}{\pi} \frac{7 \zeta(3)}{8} \bar{\Delta}^{3}\right\}+ \\
& +N_{2} V_{n 2} f_{2}\left\{\frac{N_{1} f_{1}}{N_{1} f_{1}+N_{2} f_{2}}\left(\bar{\Delta}_{1}-\bar{\Delta}_{2}\right) \ln 2 \gamma \rho^{\prime}-\frac{\beta^{2}}{8 \pi^{2}} 7 \zeta(3) \bar{\Delta}^{3}\right\}
\end{aligned}
$$


где

$$
\bar{\Delta}=\frac{N_{1} f_{1} \bar{\Delta}_{1}+N_{2} f_{2} \bar{\Delta}_{2}}{N_{1} f_{1}+N_{2} f_{2}}
$$

\section{4. ТЕМПЕРАТУРА СВЕРХПРОВОДЯЩЕГО ПЕРЕХОДА}

Вблизи $T_{C}$ представим параметры порядка $\Delta_{n}$ в виде

$$
\bar{\Delta}_{n}=c_{n} t^{\frac{1}{2}}+c_{n}^{\prime} t^{\frac{3}{2}}+\cdots, \quad t=1-\frac{T}{T_{C}}
$$

а также разложим в ряд по $t$ величины $\rho^{\prime}, \widetilde{\xi}_{n}$. Подставив эти разложения в $(21)$ и $(22)$ и приравнивая члены при $t^{1 / 2}$, получаем систему уравнений

$$
\begin{gathered}
c_{1}\left[1-N_{1} V_{11} \widetilde{\xi}_{c 1}+\frac{N_{1} N_{2} f_{1} f_{2}}{N_{1} f_{1}+N_{2} f_{2}}\left(V_{11}-V_{12}\right) F\left(\rho_{c}^{\prime}\right)\right]+ \\
+c_{2}\left[-N_{2} V_{12} \widetilde{\xi}_{c 2}-\frac{N_{1} N_{2} f_{1} f_{2}}{N_{1} f_{1}+N_{2} f_{2}}\left(V_{11}-V_{12}\right) F\left(\rho_{c}^{\prime}\right)\right]=0 \\
c_{1}\left[-N_{1} V_{21} \widetilde{\xi}_{c 1}-\frac{N_{1} N_{2} f_{1} f_{2}}{N_{1} f_{1}+N_{2} f_{2}}\left(V_{22}-V_{21}\right) F\left(\rho_{c}^{\prime}\right)\right]+ \\
+c_{2}\left[1-N_{2} V_{22} \widetilde{\xi}_{c 2}+\frac{N_{1} N_{2} f_{1} f_{2}}{N_{1} f_{1}+N_{2} f_{2}}\left(V_{22}-V_{21}\right) F\left(\rho_{c}^{\prime}\right)\right]=0
\end{gathered}
$$

здесь

$$
F\left(\rho_{c}^{\prime}\right)=\left\{\begin{array}{lll}
\frac{\pi^{2}}{4} \rho_{c}^{\prime} & \text { при } \rho_{c}^{\prime} \ll 1, \\
\ln 2 \gamma \rho_{c}^{\prime} & \text { при } \quad \rho_{c}^{\prime} \gg 1,
\end{array} \quad \rho_{c}^{\prime}=\left.\rho^{\prime}\right|_{T=T_{C}} .\right.
$$

Уравнение для определения величины $T_{C}$ получим из условия разрешимости системы (24). Приравнивая нулю определитель этой системы, получаем

$$
\begin{aligned}
a \widetilde{\xi}_{c 1} \widetilde{\xi}_{c 2} & -\widetilde{\xi}_{c 1}\left[N_{1} V_{11}+\frac{N_{1} f_{1} f_{2}}{N_{1} f_{1}+N_{2} f_{2}} a F\left(\rho_{c}^{\prime}\right)\right]- \\
& -\widetilde{\xi}_{c 2}\left[N_{2} V_{22}+\frac{N_{2} f_{1} f_{2}}{N_{1} f_{1}+N_{2} f_{2}} a F\left(\rho_{c}^{\prime}\right)\right]+ \\
& +1+\frac{N_{1} N_{2} f_{1} f_{2}}{N_{1} f_{1}+N_{2} f_{2}}\left(V_{11}-V_{12}+V_{22}-V_{21}\right) F\left(\rho_{c}^{\prime}\right)=0
\end{aligned}
$$

где

$$
a=N_{1} N_{2}\left(V_{11} V_{22}-V_{12} V_{21}\right)
$$

Решения уравнения (26) сушественно зависят от значений химического потенциала (плотности носителей заряда). Мы будем рассматривать определенные области значений химического потенщиала, когда $\left|D_{c n}\right| / T_{C},\left|D_{n}\right| / T_{C}$ значительно больше единицы, 
а также точки $D_{n}=0$ и $D_{c n}=0(n=1,2)$. Для этих случаев значения функций $f_{n}$ и $\widetilde{\xi}_{\text {cn }}$ приведены в таблице.

\begin{tabular}{|c|c|c|c|c|c|}
\hline & $\mu$ & $f_{1}$ & $f_{2}$ & $\tilde{\xi}_{1}(T)$ & $\tilde{\xi}_{2}(T)$ \\
\hline 1 & $\mu=\zeta_{1}^{\prime}$ & $1 / 2$ & 0 & $\ln \frac{2 \gamma D_{c 1}}{\pi T}$ & $\frac{1}{2} \ln \frac{D_{c 2}}{\left|D_{2}\right|}$ \\
\hline 2 & $\zeta_{1}^{\prime}<\mu<\zeta_{2}^{\prime}$ & 1 & 0 & $\ln \frac{2 \gamma \sqrt{D_{1} D_{c 1}}}{\pi T}$ & $\frac{1}{2} \ln \frac{D_{c 2}}{\left|D_{2}\right|}$ \\
\hline 3 & $\mu=\zeta_{2}^{\prime}$ & 1 & $1 / 2$ & $\ln \frac{2 \gamma \sqrt{D_{1} D_{c 1}}}{\pi T}$ & $\ln \frac{2 \gamma D_{c 2}}{\pi T}$ \\
\hline 4 & $\zeta_{2}^{\prime}<\mu<\zeta_{c 1}^{\prime}$ & 1 & 1 & $\ln \frac{2 \gamma \sqrt{D_{1} D_{c 1}}}{\pi T}$ & $\ln \frac{2 \gamma \sqrt{D_{2} D_{c 2}}}{\pi T}$ \\
\hline 5 & $\mu=\zeta_{c 1}^{\prime}$ & $1 / 2$ & 1 & $\ln \frac{2 \gamma D_{1}}{\pi T}$ & $\ln \frac{2 \gamma \sqrt{D_{2} D_{c 2}}}{\pi T}$ \\
\hline 6 & $\zeta_{c 1}^{\prime}<\mu<\zeta_{c 2}^{\prime}$ & 0 & 1 & $\frac{1}{2} \ln \frac{D_{1}}{\left|D_{c 1}\right|}$ & $\ln \frac{2 \gamma \sqrt{D_{2} D_{c 2}}}{\pi T}$ \\
\hline 7 & $\zeta_{c 2}^{\prime}<\mu$ & 0 & 0 & $\frac{1}{2} \ln \frac{D_{1}}{\left|D_{c 1}\right|}$ & $\frac{1}{2} \ln \frac{D_{2}}{\left|D_{c 2}\right|}$ \\
\hline
\end{tabular}

Ниже мы приводим значения $T_{C}$, полученные в результате решения $(26)$ для малых и больших концентраций примеси (см. таблицу):

1) $\mu=\zeta_{1}^{\prime}$ :

$$
T_{C}=\bar{T}_{c 0}=\frac{2 \gamma D_{c 1}}{\pi} \exp \left\{-2 \frac{1-N_{2} V_{22} \frac{1}{2} \ln \frac{D_{c 2}}{-D_{2}}}{N_{1} V_{11}-a \frac{1}{2} \ln \frac{D_{c 2}}{-D_{2}}}\right\} ;
$$

2) $\zeta_{1}^{\prime}<\mu<\zeta_{2}^{\prime}$

$$
T_{C}=\bar{T}_{c 0}=\frac{2 \gamma \sqrt{D_{1} D_{c 1}}}{\pi} \exp \left\{-\frac{1-N_{2} V_{22} \frac{1}{2} \ln \frac{D_{c 2}}{-D_{2}}}{N_{1} V_{11}-a \frac{1}{2} \ln \frac{D_{c 2}}{-D_{2}}}\right\}
$$

3) $\mu=\zeta_{2}^{\prime}$ :

$$
\begin{array}{ll}
T_{C}=\bar{T}_{c 0}^{\frac{1}{1-\alpha}}\left[\frac{\gamma}{\pi \tau_{12}}\left(\frac{1}{2}+\frac{\tau_{12}}{\tau_{21}}\right)\right]^{\frac{\alpha}{\alpha-1}} \quad \text { при } \rho_{n} \gg 1, \\
T_{C}=\bar{T}_{c 0}-\alpha \frac{\pi}{8 \tau_{12}}\left(\frac{1}{2}+\frac{\tau_{12}}{\tau_{21}}\right) & \text { при } \rho_{n} \ll 1,
\end{array}
$$

где

$$
\begin{aligned}
\bar{T}_{c 0}= & \frac{2 \gamma\left(D_{1} D_{c 1}\right)^{\frac{1}{4}} D_{c 2}^{\frac{1}{2}} \exp \left\{-\frac{2 N_{1} V_{11}+N_{2} V_{22}}{2 a} \mp\right.}{\pi} \\
& \left.\mp \frac{1}{4 a}\left[\left(a \ln \frac{D_{1} D_{c 1}}{D_{c 2}^{2}}+4 N_{1} V_{11}-2 N_{2} V_{22}\right)^{2}+32 N_{1} N_{2} V_{12} V_{21}\right]^{\frac{1}{2}}\right\}
\end{aligned}
$$




$$
\begin{aligned}
\alpha= & \frac{1}{2}\left\{1 \pm \frac{1}{\sqrt{b_{0}^{2}-4 a c_{0}}}\left[N_{1} V_{11} \frac{2 N_{1}-N_{2}}{2 N_{1}+N_{2}}+\right.\right. \\
& \left.\left.+\frac{1}{2} N_{2} V_{22} \frac{N_{2}-2 N_{1}}{2 N_{1}+N_{2}}+\frac{2 N_{1} N_{2}}{2 N_{1}+N_{2}}\left(V_{12}+V_{21}\right)\right]\right\}, \\
b_{0}= & N_{1} V_{11}+\frac{1}{2} N_{2} V_{22}+\frac{a}{2} \ln \frac{\sqrt{D_{1} D_{c 1}}}{D_{c 2}} \\
c_{0}= & 1-\frac{1}{2} N_{2} V_{22} \ln \frac{\sqrt{D_{1} D_{c 1}}}{D_{c 2}}
\end{aligned}
$$

4) $\zeta_{2}^{\prime}<\mu<\zeta_{c 1}^{\prime}$ :

$$
\begin{array}{lll}
T_{C}=\bar{T}_{c 0}-\alpha \frac{\pi}{8 \tau_{12}}\left(1+\frac{\tau_{12}}{\tau_{21}}\right) & \text { при } & \rho_{n} \ll 1, \\
T_{C}=\bar{T}_{c 0}^{\frac{1}{1-\alpha}}\left[\frac{\gamma}{\pi \tau_{12}}\left(1+\frac{\tau_{12}}{\tau_{21}}\right)\right]^{\frac{\alpha}{\alpha-1}} \text { при } & \rho_{n} \gg 1,
\end{array}
$$

здесь

$$
\begin{aligned}
\bar{T}_{c 0}= & \frac{2 \gamma\left(D_{1} D_{c 1} D_{2} D_{c 2}\right)^{\frac{1}{4}}}{\pi} \exp \left\{-\frac{N_{1} V_{11}+N_{2} V_{22}}{2 a} \mp\right. \\
& \left.\mp \frac{1}{2 a}\left[\left(a \ln \frac{D_{1} D_{c 1}}{D_{2} D_{c 2}}+N_{1} V_{11}+N_{2} V_{22}\right)^{2}+4 N_{1} N_{2} V_{12} V_{21}\right]^{\frac{1}{2}}\right\}, \\
\alpha= & \frac{1}{2}\left\{1 \pm \frac{1}{\sqrt{b_{0}^{2}-4 a c_{0}}}\left[N_{1} V_{11} \frac{N_{1}-N_{2}}{N_{1}+N_{2}}+\right.\right. \\
& \left.\left.+N_{2} V_{22} \frac{N_{2}-N_{1}}{N_{1}+N_{2}}+\frac{2 N_{1} N_{2}}{N_{1}+N_{2}}\left(V_{12}+V_{21}\right)\right]\right\}, \\
b_{0}= & N_{1} V_{11}+N_{2} V_{22}+a \ln \frac{D_{2} D_{c 2}}{D_{1} D_{c 1}}, \\
c_{0}= & 1-N_{2} V_{22} \ln \frac{D_{2} D_{c 2}}{D_{1} D_{c 1}} ;
\end{aligned}
$$

5) $\mu=\zeta_{c 1}^{\prime}$ :

$$
\begin{aligned}
& T_{\mathrm{C}}=\bar{T}_{c 0}-\alpha \frac{\pi^{2}}{8 \tau_{12}}\left(1+\frac{\tau_{12}}{2 \tau_{21}}\right) \quad \text { при } \rho_{n} \ll 1, \\
& T_{\mathrm{C}}=\bar{T}_{c 0}^{\frac{1}{1-\alpha}}\left[\frac{\gamma}{\pi \tau_{12}}\left(1+\frac{\tau_{12}}{2 \tau_{21}}\right)\right]^{\frac{\alpha}{\alpha-1}} \text { при } \rho_{n} \gg 1,
\end{aligned}
$$


где

$$
\begin{aligned}
\bar{T}_{c 0}= & \frac{2 \gamma \sqrt{D_{1}}\left(D_{2} D_{c 2}\right)^{\frac{1}{4}}}{\pi} \exp \left\{-\frac{N_{1} V_{11}+2 N_{2} V_{22}}{2 a} \mp\right. \\
& \left.\mp \frac{1}{2 a}\left[\left(a \ln \frac{D_{1}}{\sqrt{D_{2} D_{c 2}}}+N_{1} V_{11}-2 N_{2} V_{22}\right)^{2}+8 N_{1} N_{2} V_{12} V_{21}\right]^{\frac{1}{2}}\right\}, \\
\alpha= & \frac{1}{2}\left\{1 \pm \frac{1}{\sqrt{b_{0}^{2}-4 a c_{0}}}\left[\frac{N_{1} V_{11}}{2} \frac{N_{1}-2 N_{2}}{N_{1}+2 N_{2}}+\right.\right. \\
& \left.\left.+N_{2} V_{22} \frac{2 N_{2}-N_{1}}{N_{1}+2 N_{2}}+\frac{2 N_{1} N_{2}}{N_{1}+2 N_{2}}\left(V_{12}+V_{21}\right)\right]\right\}, \\
b_{0}= & \frac{N_{1} V_{11}}{2}+N_{2} V_{22}-\frac{a}{2} \ln \frac{\sqrt{D_{2} D_{c 2}}}{D_{1}} \\
c_{0}= & 1-N_{2} V_{22} \ln \frac{\sqrt{D_{2} D_{c 2}}}{D_{1}}
\end{aligned}
$$

6) $\zeta_{c 1}^{\prime}<\mu<\zeta_{c 2}^{\prime}$ :

$$
T_{C}=\bar{T}_{c 0}=\frac{2 \gamma \sqrt{D_{2} D_{c 2}}}{\pi} \exp \left\{-\frac{2+N_{1} V_{11} \ln \frac{-D_{c 1}}{D_{1}}}{2 N_{2} V_{22}+a \ln \frac{-D_{c 1}}{D_{1}}}\right\} ;
$$

7) $\zeta_{c 2}^{\prime}<\mu: T_{C}=0$.

При $1 / \tau_{n m}=0$ имеем $T_{C}=T_{c 0}$. Выражения для $T_{c 0}$ совпадают с полученными нами ранее [17] и описывают зависимость температуры сверхпроводящего перехода от плотности носителей заряда (химического потенциала) в упорядоченных системах. Эта зависимость представляется в виде несколько деформированного колокола. При этом степень деформации определяется значениями констант внутризонного и межзонного взаимодействий $\left[17\right.$, рис. 1]. Формулы (33) и (34) при $D_{1}=D_{c 1}=D_{2}=D_{c 2}=\omega_{D}$ совпадают с полученными нами ранее [4] и отвечают температуре сверхпроводящего перехода в обычных двухзонных примесных сверхпроводниках с малой и большой концентрациями примеси, соответственно.

Нетрудно видеть, что приведенные выше выражения для $T_{C}$ указьвают на сушественную роль фактора заполнения энергетических зон в случае нефононного механизма сверхпроводимости. В самом деле, выражения (27), (28) и (40) не содержат примесной зависимости (за исключением перенормировки величин $D_{n}$ и $D_{c n}$ ). В остальных же формулах как в случае малых, так и больших концентраций примеси эта зависимость содержится за счет наличия времен релаксации $\tau_{n m}$ межзонного рассеяния на примеси. При этом для различных $\mu$ мы имеем разные значения не только величин $T_{c 0}$, но и коэффициентов, определяющих примесную зависимость.

Полученные выражения для $T_{C}$ можно попытаться использовать для описания зависимости температуры сверхпроводяшего перехода от плотности носителей заряда, например в иттриевой керамике $\mathrm{YBa}_{2} \mathrm{Cu}_{3} \mathrm{O}_{7-\delta}$. Мы полагаем, что металлическая фаза в этом соединении с максимальной температурой сверхпроводящего перехода $T_{C} \approx 90 \mathrm{~K}$ 
устанавливается при $\delta=0\left(\mu=\mu_{\max }\right)$. С уменьшением количества кислорода (увеличением $\delta$ ) происходит разупорядочение системы, возникают хаотически распределенные кислородные вакансии, на которых происходит рассеяние электронов. Картина аналогична хаотически распределенной примеси. Убывание $T_{C}$ вызвано двумя факторами: уменьшением химического потенциала и межзонным рассеянием электронов на кислородных вакансиях. Убывание величины $T_{C}$ в области $\mu<\mu_{\max }$ будет происходить более быстрыми темпами, чем в случае неучета межзонного рассеяния на вакансиях кислорода [17].

Развитая здесь теория может быть применима также для описания влияния примеси, заменяюшей $\mathrm{Cu}$ в $\mathrm{YBa}_{2} \mathrm{Cu}_{3} \mathrm{O}_{7-\delta}$ при фиксированных значениях $\delta$. В случае упорядоченных по кислороду состояний основным механизмом, способствуюшим убыванию $T_{C}$ с ростом примеси, выступает межзонное рассеяние на примеси. Мы говорим здесь об иттриевой керамике, в которой твердо установленным фактом является перекрытие энергетических зон на поверхности Ферми [24]. Такая же ситуация имеет место и в более сложных материалах ВТСП.

Приведенные выше формулы содержат и другие возможности. В частности, если предположить, что металлическая фаза в примесном сверхпроводнике устанавливается при $\mu<\mu_{\max }$, а примесь увеличивает химический потенциал, то в определенной области концентраций примеси будет происходить конкуренция механизма роста $T_{C}$ с ростом $\mu$ и механизма убывания $T_{C}$ из-за межзонного рассеяния на примеси. В результате может оказаться возможным рост $T_{C}$ с ростом концентраций примеси.

Важной проблемой в настояшее время является выявление роли примеси цинка, замещающего атомы меди в материалах ВТСП. Цинк, будучи немагнитной примесью, ведет себя как магнитная, подавляя сверхпроводимость. В рамках предложенной выше теории можно понять исчезновение сверхпроводимости при введении немагнитной примеси. В самом деле, если введение примеси приводит к росту химического потенциала, то при $\mu>\mu_{\max }$ происходит убывание величины $T_{C}$ из-за роста $\mu$ и межзонного рассеяния на примеси. При этом в области значений $\zeta_{c 2}^{\prime}<\mu$ величина $T_{C}$ равна нулю за счет фактора заполнения энергетических зон и выхода химического потенциала за пределы рассматриваемых энергетических зон.

\section{5. ПАРАМЕТРЫ ПОРЯДКА ПРИ $T=0$}

Система уравнений на параметры порядка при $T=0$ на основании (11) может быть представлена в виде

$$
\bar{\Delta}_{n}=\sum_{l} V_{n l} N_{l} \bar{\Delta}_{l} \xi_{l}(0)+\sum_{l} V_{n l} N_{l} \frac{2 \pi}{\beta} \sum_{\omega>0} f_{l}\left(\frac{1}{\sqrt{u_{l}^{2}+1}}-\frac{\bar{\Delta}_{l}}{\sqrt{\omega^{2}+\bar{\Delta}_{l}^{2}}}\right)
$$

где

$$
\xi_{l}(0)=\frac{1}{2} \int_{-D_{l}}^{D_{c l}} \frac{d \tilde{\varepsilon}_{l}}{\sqrt{\tilde{\varepsilon}_{l}^{2}+\bar{\Delta}_{l}^{2}}} .
$$

Здесь мы будем рассматривать большие концентрации примеси, при этом

$$
\alpha_{1}, \alpha_{2} \gg 1, \quad u_{l}=\frac{\widetilde{\omega}_{l}}{\widetilde{\Delta}_{l}} \gg 1
$$


и величины $u_{l}$ удовлетворяют системе уравнений $(17)$. Подставив в $(14)$ решения $(18)$ и выполнив интегрирование по $\omega(T=0)$, получаем [5]

$$
\bar{\Delta}_{n}=\sum_{l} V_{n l} N_{l} \bar{\Delta}_{l} \xi_{l}(0)+\sum_{l} V_{n l} N_{l} f_{l} \bar{\Delta}_{l} \varphi_{l}
$$

где

$$
\begin{aligned}
\varphi_{1}= & \frac{\alpha_{1} f_{2}(1-\bar{z})}{\alpha_{2} f_{1}+\alpha_{1} f_{2} \bar{z}} \ln \left[2\left(\alpha_{1} f_{2}+\alpha_{2} f_{1} / \bar{z}\right)\right]+ \\
& +\frac{\alpha_{2} f_{1}+\alpha_{1} f_{2}}{\alpha_{2} f_{1}+\alpha_{1} f_{2} \bar{z}} \ln \frac{\alpha_{2} f_{1}+\alpha_{1} f_{2} \bar{z}}{\alpha_{2} f_{1}+\alpha_{1} f_{2}}, \quad \bar{z}=\frac{\bar{\Delta}_{1}}{\bar{\Delta}_{2}},
\end{aligned}
$$

$\varphi_{2}$ получим из $\varphi_{1}$ заменой индексов $1 \rightleftarrows 2, \bar{z} \rightarrow 1 / \bar{z}$.

Приведем решения уравнения (42) в наиболее интересной области значений химического потенциала. При $\zeta_{2}^{\prime} \leq \mu \leq \zeta_{c 1}^{\prime}$ (область значений 3-5 таблицы) параметр порядка $\Delta_{1}$ определяется выражением

$$
\bar{\Delta}_{1}=2 \sqrt{D_{1} D_{c 1}} e^{-\xi_{1}}
$$

где

$$
\xi_{1}=\frac{N_{2} V_{22}-\frac{1}{\bar{z}} N_{2} V_{12}}{a}-\varphi_{1}(\bar{z}) f_{1},
$$

а $\varphi_{1}$ дано формулой (43), в которой $f_{n}$ определяется из таблицы в соответствии со значением величины $\mu$. В этом случае $\bar{z}$ удовлетворяет уравнениям:

при $\mu=\zeta_{2}^{\prime}$ (п. 3 таблицы) -

$$
\begin{aligned}
\frac{1}{2} a \ln \bar{z}= & N_{1}\left(V_{11}-V_{21} \bar{z}\right)-\frac{1}{2} N_{2}\left(V_{22}-V_{12} / \bar{z}\right)- \\
& -\frac{1}{2} a \ln \frac{D_{c 2}}{\sqrt{D_{1} D_{c 1}}}-\frac{1}{2} a\left(\frac{1}{2} \varphi_{2}-\varphi_{1}\right)
\end{aligned}
$$

при $\zeta_{2}^{\prime}<\mu<\zeta_{c 1}^{\prime}($ п. 4 таблицы $)-$

$$
\begin{aligned}
a \ln \bar{z}= & N_{1}\left(V_{11}-V_{21}\right) \bar{z}-N_{2}\left(V_{22}-V_{12} / \bar{z}\right)- \\
& -a \ln \frac{\sqrt{D_{2} D_{c 2}}}{D_{1} D_{c 1}}-a\left(\varphi_{2}-\varphi_{1}\right)
\end{aligned}
$$

при $\mu=\zeta_{c 1}^{\prime}($ п. 5 таблицы $)-$

$$
\begin{aligned}
\frac{1}{2} a \ln \bar{z}= & \frac{1}{2} N_{1}\left(V_{11}-V_{21} \bar{z}\right)-N_{2}\left(V_{22}-V_{12} / \bar{z}\right)- \\
& -\frac{1}{2} a \ln \frac{\sqrt{D_{2} D_{c 2}}}{D_{1}}+\frac{1}{2} a\left(\frac{1}{2} \varphi_{1}-\varphi_{2}\right) .
\end{aligned}
$$

Нетрудно видеть на основании (46)-(48), что величина $\bar{z}$ сушественно зависит от значения химического потенциала. Для выявления зависимости $\Delta_{n}$ от значения химического 
потенциала и концентрации примеси необходимо найти решения для $\bar{z}$ и использовать выражения (44) и (45).

В приближении слабой связи можно решить уравнения (46)-(48) и получить выражения для параметров порядка $\Delta_{1}$ и $\Delta_{2}$. Запишем эти уравнения единым образом:

$$
a^{\prime} \ln z=\widetilde{N_{1}}\left(V_{11}-V_{21} \bar{z}\right)-\widetilde{N_{2}}\left(V_{22}-V_{12} / \bar{z}\right)-a^{\prime} \ln Q+a^{\prime}\left(f_{1} \varphi_{1}-f_{2} \varphi_{2}\right),
$$

где

$$
\tilde{N}_{n}=N_{n} f_{n}, \quad a^{\prime}=f_{1} f_{2} a, \quad Q= \begin{cases}D_{2} / \sqrt{D_{1} D_{c 1}} & \text { при } \mu=\zeta_{2}^{\prime}, \\ \left(D_{2} D_{c 2} / D_{1} D_{c 1}\right)^{\frac{1}{2}} & \text { при } \zeta_{2}^{\prime} \leq \mu \leq \zeta_{c 1}^{\prime}, \\ \left(D_{2} D_{c 2}\right)^{\frac{1}{2}} / D_{1} & \text { при } \mu=\zeta_{c 1}^{\prime} ;\end{cases}
$$

$f_{1}$ и $f_{2}$ даны в таблице.

Ищем решение (49) в виде

$$
\bar{z}=z_{0}+z^{\prime}
$$

где $z_{0}$ удовлетворяет уравнению

$$
\widetilde{N}_{1} V_{11}-\widetilde{N}_{2} V_{22}-\widetilde{N}_{1} V_{21} z_{0}+\widetilde{N}_{2} V_{12} / z_{0}=0
$$

Подставляя (51) в (49) и ограничиваясь квадратичными по константам взаимодействия членами, получаем

$$
z^{\prime}=-a^{\prime} \ln \left\{z_{0} Q\left[F_{2}\left(z_{0}\right)\right]^{f_{2}} /\left[F_{1}\left(z_{0}\right)\right]^{f_{1}}\right\}\left[\tilde{N}_{2} V_{22}+\frac{\widetilde{N}_{2} V_{12}}{z_{0}^{2}}\right]^{-1}
$$

где

$$
\ln F_{n}\left(z_{0}\right)=\varphi_{n}\left(z_{0}\right) .
$$

Используя определения (44) и (45), находим

$$
\bar{\Delta}_{1}=\bar{\Delta}_{1}^{0}\left[F_{2}\left(z_{0}\right)\right]^{\gamma f_{2}}\left[F_{1}\left(z_{0}\right)\right]^{f_{1}(1-\gamma)}, \quad \bar{\Delta}_{2}=\bar{\Delta}_{1} / \bar{z}
$$

где

$$
\begin{gathered}
\Delta_{1}^{0}=2 \sqrt{D_{1} D_{c 1}}\left[z_{0} Q\right]^{\gamma} \exp \left(-\frac{N_{2} V_{22}-N_{2} V_{12} / z_{0}}{a}\right), \\
\gamma=\frac{f_{1} \widetilde{N}_{2}}{\widetilde{N}_{1}+\widetilde{N}_{2} / z_{0}^{2}} .
\end{gathered}
$$

Таким образом, если рассматривать изовалентную примесь, введение которой в систему не влияет на химический потенциал, то при фиксированном $\mu$ величина $\Delta_{1}^{0}$ будет определять значение параметра порядка для чистого вешества, а остальные множители в (55) - примесную зависимость, поскольку величины $F_{1}$ и $F_{2}$ содержат степенную зависимость от концентрации примеси. Если же вводится примесь, которая меняет значение химического потенциала, то ее влияние на параметры порядка $\Delta_{1}$ и $\Delta_{2}$ определяется двумя механизмами, а именно изменением химического потенциала (концентрации 
носителей) и межзонным рассеянием электронов на примесном потенциале. При этом изменяется как $\bar{\Delta}_{1}^{0}$, так и явная зависимость $\bar{\Delta}_{1}$ от концентрации примеси с изменением значения химического потенциала. Величина $\Delta_{1}^{0}$ как функция от $\mu$ приведена на рис. 3 работы [17]. С ростом $\mu$ происходит рост величины $\Delta_{1}^{0}$, а затем ее убывание. Такоеповедение объясняется фактором последовательного заполнения энергетических зон. Учет межзонного рассеяния на примеси приводит к понижению $\bar{\Delta}_{1}$ по сравнению с $\bar{\Delta}_{1}^{0}$. Как и в случае величины $T_{C}$ (см. выше), имеются различные возможности описания зависимости $\bar{\Delta}_{n}$ от концентрации примеси в различных системах.

\section{6. ЗАКЛЮЧЕНИЕ}

В данной работе была поставлена задача исследовать влияние примеси на температуру сверхпроводящего перехода $T_{C}$ и параметры порядка $\Delta_{1}$ и $\Delta_{2}$ в системе с двумя перекрывающимися энергетическими зонами на поверхности Ферми при нефононном механизме сверхпроводимости. На основании теории возмущений [22] и метода функций Грина $[5,23]$ получены основные уравнения теории сверхпроводимости $(11),(12)$ для неупорядоченной двухзонной системы. Рассмотрена область температур $T=0$ и $T \sim T_{C}$ и случаи больших и малых концентраций примеси.

Уравнение для температуры сверхпроводяшего перехода (26) решено для конкретных заданных значений химического потенциала $\mu$ (формулы (27)-(40)). Эти аналитические формулы позволяют описать зависимость $T_{C}$ от $\mu$ при заданных концентрациях примеси и зависимость $T_{C}$ от концентрации примеси при заданном $\mu$ в области больших и малых концентраций примеси. Полученная зависимость содержит большие возможности для объяснения имеющихся экспериментальных данных. Мы отмечаем два механизма, способствующих изменению величины $T_{C}$ при введении замещающей примеси другой валентности в сверхпроводяшую систему: изменение химического потенциала $\mu$ (плотности носителей заряда) и возникновение межзонного рассеяния электронов на примеси. Влияние этих двух механизмов на возникновение или разрушение сверхпроводимости зависит от тех значений $\mu$, при которых устанавливается металлическая фаза с высокими $T_{C}$ в системе. Поскольку данная теория дает зависимость $T_{C}$ от $\mu$ в виде деформированного колокола с максимумом $T_{C}$ в точке $\mu=\mu_{\max }$, а межзонное рассеяние приводит к убыванию $T_{C}$, то при $\mu<\mu_{\max }$ вышеуказанные механизмы являются конкурируюшими, а при $\mu>\mu_{\max }$ оба способствуют убыванию $T_{C}$ с ростом $\mu$. При этом возможно полное разрушение сверхпроводимости немагнитной примесью $\left(T_{C}=0\right)$, как это наблюдается в купратных соединениях при введении цинка вместо меди.

Слабое влияние примесей $\mathrm{Al}$ и Ga в купратных соединениях можно объяснить отсутствием этого влияния на $\mu$ и слабым межзонным рассеянием, поскольку эти примеси замещают атомы меди вне плоскости $\mathrm{CuO}$, ответственной за сверхпроводимость.

Нельзя утверждать, что полученные результаты по зависимости $T_{C}$ от конщентрации примеси решают проблему влияния примеси на величину $T_{C}$ в высокотемпературных сверхпроводниках. Однако предложенная выше теория позволяет объяснить экспериментальные данные.

Мы получили также аналитические выражения для параметров порядка $\bar{\Delta}_{1}$ и $\bar{\Delta}_{2}(55)$ в случае определенных значений химического потенциала и выявили явную степенную зависимость этих параметров от концентрации примеси в области больших значений последней. При этом величина степенного показателя наряду с другими пара- 
метрами двухзонной теории зависит от фактора заполнения энергетических зон (включения и отключения соответствующих энергетических зон с изменением химического потенциала).

\section{Список литературы}

[1] P. W. Anderson. J. Phys. Chem. Solids. 1959. V. 11. P. 26.

[2] А.А. Абрикосов, Л. П. Горьков. ЖЭТФ. 1960. Т. 39. С. 1781.

[3] D. Marcowitz, L.P. Kadanoff. Phys. Rev. 1963. V. 131. P. 563.

[4] В. А. Москаленко, М. Е. Палистрант. ДАН СССР. 1965. Т. 162. С. 539; ЖЭЭФ. 1965. T. 49. C. 770 .

[5] В.А. Москаленко, Л. З. Кон, М.Е. Палистрант. Низкотемпературные свойства металлов с особенностями зонного спектра. Кишинев: Штиинца, 1989.

[6] A. A. Abricosov, J. C. Camuzano, H. Gofron. Physica C. 1993. V. 214. P. 73.

[7] М. Е. Палистрант, А.Т. Трифан. ФНТ. 1977. Т. 3. С. 241, 976.

[8] М. Е. Палистрант, О. П. Беззуб. ФНТ. 1980. Т. 6. С. 1146; 1983. Т. 9. С. 357.

[9] J. G. Bednorz, K. A. Muller. Z. Phys. B. 1986. B. 64. S. 189.

[10] V. A. Moscalenco, M. E. Palistrant, V. M. Vakalyuk. Solid State Commun. 1989. V. 69. Р. 747; В. А. Москаленко, М. Е. Палистрант, В. М. Вакалюк. УФН. 1991. Т. 161. С. 155.

[11] В. А. Москаленко. ФММ. 1959. Т. 8. С. 504.

[12] H. Suhl, B. T. Mathias, L. K. Walker. Phys. Rev. Lett. 1959. V. 3. P. 552.

[13] I. Grosu, I. Veres, M. Crisan. Phys. Rev. B. 1994. V. 50. № 1. P. 9404.

[14] I. Grosu. J. of Superconductivity. 1996. V. 9. P. 113.

[15] R. E. Walstedt, R.F. Bell, L.F. Schneermeyer, I. V. Waszczak. Phys. Rev. B. 1993. V. 48. № 2. P. 10646.

[16] P. Konsin, N. Kristoffel, T. Ord. Phys. Lett. 1988. V. 129. P. 399.

[17] M. E. Palistrant, F. G. Kochorbe. Physica C. 1992. V. 198. P. 351.

[18] Ф. Г. Кочорбә, М. Е. Палистрант. ЖЭТФ. 1993. Т. 104. С. 3084; ТМФ. 1993. Т. 96. C. 459.

[19] Э. В. Горбар, В. П. Гусынин, В. М. Локтев. Сверхпроводимость: ФХТ. 1993. Т. 6. С. 483; ФHT. 1993. T. 19. C. 1171.

[20] М. Е. Палистрант, В. М. Вакалюк. Сверхпроводимость: ФХТ. 1990. Т. 3. Ч. 2. С. 1805.

[21] М. Г. Калалб, Ф.Г. Кочорбә, М. Е. Палистрант. ТМФ. 1992. Т. 91. С. 483.

[22] А.А. Абрикосов, Л. П. Горьков, И. Е. Дзялошинский. Методы квантовой теории поля в статистической физике. М.: Наука, 1962.

[23] В. А. Москаленко. ДАН СССР. 1967. Т. 176. С. 301; ФММ. 1967. Т. 23. С. 585.

[24] J.F. Herman, R. W. Kasowski, W. Y. Hsau. Phys. Rev. B. 1987. V. 36. P. 6904.

Поступила в редакцию 16.XII.1996 г.

\section{M.E. Palistrant \\ INFLUENCE OF NONMAGNETIC ADMIXTURE ON SUPERCONDUCTIVITY IN TWO-ZONE SYSTEMS WITH VARIABLE DENSITY OF CHARGE CARRIERS}

By perturbation theory the analytical expressions for the temperature of the superconductivity transition $T_{C}$ and order parameters $\Delta_{n}(n=1,2)$ under different values of density of charge carriers (chemical potential $\mu$ ) are obtained for two-zone superconductors with nonmagnetic admixture. When admixture is input two mechanisms of its influence on $T_{C}$ and $\Delta_{n}$ are possible: the change of $\mu$ and the appearance of interzone scattering on the admixture potential. These mechanisms can compensate each other or both promote the destruction of superconductivity. This essentially depends on the role played by the factor of filling the energy zones due to which superconductivity can be completely suppressed by a nonmagnetic admixture. 